\title{
$q$-LAGUERRE POLYNOMIALS AND BIG $q$-BESSEL FUNCTIONS AND THEIR ORTHOGONALITY RELATIONS
}

\author{
Nicola Ciccoli, Erik Koelink, and Tom H. Koornwinder \\ Dedicated to Richard Askey on the occasion of his 65th birthday
}

\begin{abstract}
The $q$-Laguerre polynomials correspond to an indeterminate moment problem. For explicit discrete non- $\mathrm{N}$-extremal measures corresponding to Ramanujan's $1 \psi_{1}$-summation, we complement the orthogonal $q$-Laguerre polynomials to an explicit orthogonal basis for the corresponding $L^{2}$-space. The dual orthogonal system consists of so-called big $q$-Bessel functions, which can be obtained as a rigorous limit of the orthogonal system of big $q$-Jacobi polynomials. Interpretations on the $S U(1,1)$ and $E(2)$ quantum groups are discussed.
\end{abstract}

\section{Introduction}

This paper answers two seemingly different questions which both originated from quantum groups. First, consider the system of Moak's [21] $q$-Laguerre polynomials with respect to their familiar discrete orthogonality measure. Can we extend this in an explicit way to a complete orthogonal system of eigenfunctions of a doubly infinite Jacobi matrix originating from analysis on $S U_{q}(1,1)$, the quantum $S U(1,1)$ group? Second, can we obtain orthogonality relations and dual orthogonality relations for certain (big) $q$-Bessel functions originating (see [4]) on $E_{q}(2)$, the quantum group of plane motions, and give rigorous proofs of these orthogonalities? The two questions are related because the dual orthogonality relations for the big $q$-Bessel functions turn out to be the orthogonality relations for the completed $q$-Laguerre polynomials.

It is well known that the moment problem corresponding to Moak's $q$-Laguerre polynomials is indeterminate as a Stieltjes moment problem. Moak [21, Theorems 1 and 2] gives several orthogonality measures for the $q$-Laguerre polynomials: an absolutely continuous measure on $[0, \infty)$ and purely discrete measures supported on the set $\left\{c q^{k} \mid k \in \mathbb{Z}\right\}$ for any constant $c>0$; see $\S 4$ for the explicit weights. See also Ismail and Rahman [10] for the explicit calculation of the entire functions in the Nevanlinna parametrization of the orthogonality measures for the moment problem for the $q$-Laguerre polynomials. We are interested in the discrete orthogonality measures. From the general theory of orthogonal polynomials, it follows that the polynomials are not dense in the corresponding space of quadratically integrable functions since the support is not the set of zeros of an entire function. This is expressed by saying that the measure is not N-extremal. See [1] for more information on moment problems and orthogonal polynomials. In this paper, we complement the $q$-Laguerre

Received April 24, 1998, revised September 10, 1998.

1991 Mathematics Subject Classification: 33D45, 33D80.

Key words and phrases: orthogonal polynomials, indetermined moment problem, non-extremal measures, $q$-Bessel functions, $S U(1,1)$ and $E(2)$ quantum groups. 
polynomials to an orthogonal basis of the $L^{2}$-space for the discrete measure by using a certain $q$-analogue of the Bessel function of order $\alpha$. The dual basis functions can be recognized as big $q$-Bessel functions; see below.

Bessel functions have $q$-analogues of several sorts. Best known and probably the oldest ones are Jackson's first and second $q$-Bessel functions; see Ismail [8]. They occur in many places, including the present paper, but they have the draw-back that they do not form an orthogonal system (possibly they form a biorthogonal system). Other $q$-analogues of Bessel functions can be obtained as formal limit cases of the three $q$-analogues of Jacobi polynomials, i.e., of little $q$-Jacobi polynomials, big $q$-Jacobi polynomials, and Askey-Wilson (AW) polynomials. For this reason, we propose to speak about little $q$-Bessel functions, big $q$-Bessel functions, and AW-type $q$-Bessel functions for the corresponding limit cases. Little, big, and AW-type $q$-Bessel functions have interpretations on $E_{q}(2)$ completely analogous to the interpretations of little and big $q$-Jacobi polynomials and Askey-Wilson polynomials on $S U_{q}(2)$, the quantum $S U(2)$ group; see Vaksman and Korogodskiı [23], Koelink [14, 15], and Bonechi et al. [4]. See also Ismail et al. [9] for the Fourier-Bessel transform of the AW-type $q$-Bessel function. The duals of the AW-type $q$-Bessel functions also can be viewed as the little $q$-Jacobi functions which live on $S U_{q}(1,1)$; see Masuda et al. [20]. These three types of $q$-Bessel functions satisfy orthogonality relations which can be obtained as formal limits of the corresponding orthogonality relations for $q$-Jacobi polynomials. In the $q$-Bessel case, it is not sufficient to give orthogonality relations; one also has to prove completeness, either directly or by giving dual orthogonality relations. For rigorous proofs of orthogonality and completeness, we mention three different techniques: (i) spectral theoretic methods, (ii) direct proofs by use of generating functions, and (iii) rigorous limit transitions from the orthogonal polynomials case. Usually, only the first method yields both orthogonality and completeness. The second method was used for little $q$-Bessel functions by Koornwinder and Swarttouw [18] and was sufficient because of self-duality. The first method was used for little $q$-Jacobi functions by Kakehi et al. $[11,12]$. In the present paper, we give three different proofs, first by the spectral method, and next by method (ii) for the extension of the $q$-Laguerre orthogonality, and then by method (iii) for the big $q$-Bessel orthogonality.

The spectral method, developed in Sections 2 and 3, is based on the spectral analysis of a doubly infinite Jacobi matrix, see e.g., Masson and Repka [19]. This operator arises in a natural way from $S U_{q}(1,1)$. The spectral analysis is very similar to the spectral analysis for second order differential equations (see [6]), such as for the second order differential equation satisfied by the Jacobi functions (see a survey of these functions in [17]). The support of the spectral measure is determined by the zeros of a $c$-function. We calculate the Green's function, and we obtain the spectral measure for the doubly infinite Jacobi matrix from the Green's function. The spectral measure is discrete and its support falls into two sets, one set corresponding to eigenvectors in terms of the $q$-Laguerre polynomials and the other set corresponding to eigenvectors in terms of the $q$-Bessel coefficients. It should be noted that this method corresponds to the one employed by Kakehi et al. [11, 12] giving the Plancherel measure for the little $q$-Jacobi functions from its interpretation as matrix elements of irreducible representations of $S U_{q}(1,1)$.

The orthogonality relations and squared norms resulting from the spectral analysis are explicitly given in $\S 4$. Since we give a basis for this space, we also obtain the dual orthogonality relations as an immediate consequence. Using a method of Berg [3], we 
can easily construct more non-N-extremal orthogonality measures for the $q$-Laguerre polynomials on the same set $\left\{c q^{k} \mid k \in \mathbb{Z}\right\}$ by perturbing with any of the $q$-Bessel functions which are bounded on this set.

In $\S 5$, we give a straightforward proof of the orthogonality relations for the $q$ Laguerre polynomials and $q$-Bessel functions using two generating functions for the $q$-Bessel functions. This technique is motivated by $[13,18]$.

In $\S 6$, we obtain the orthogonality relations for the big $q$-Bessel functions as a rigorous limit of the big $q$-Jacobi polynomial case. The formal limit is suggested by the limit transition of $S U_{q}(2)$ group to $E_{q}(2)$. Big $q$-Jacobi polynomials have an interpretation as basis elements for the regular representation of $S U_{q}(2)$ on quantum spheres; see [22]. In the limit transition, these basis elements tend to the corresponding basis elements for the regular representation of $E_{q}(2)$ on quantum hyperboloids, and these latter basis elements can be written as big $q$-Bessel functions; see [4].

So we see that the same result on $q$-special functions can be obtained from two different quantum group interpretations: by considering the quantum $S U(1,1)$ group or the quantum group of plane motions. This is also the case for the little $q$-Jacobi functions studied in $[11,12]$ which originated as spherical functions on $S U_{q}(1,1)$, but which also can be viewed as certain $q$-analogues of Bessel functions and then have an interpretation on the quantum group of plane motions; see [15].

Notation. We follow the notation of Gasper and Rahman [7] for basic (or $q$-) hypergeometric series. Throughout we assume that $0<q<1$.

\section{Solutions to a symmetric operator}

Consider the unbounded operator $L$ acting on $\ell^{2}(\mathbb{Z})$ by

$$
\begin{gathered}
(L u)_{k}=a_{k} u_{k+1}+b_{k} u_{k}+a_{k-1} u_{k-1}, \quad u=\left(u_{k}\right)_{k \in \mathbb{Z}}, \\
a_{k}=q^{-\frac{1}{2}(k+1)} \sqrt{1+c^{-1} q^{-k}}, \quad b_{k}=\sqrt{c^{-1}}\left(t+t^{-1}\right) q^{-k}
\end{gathered}
$$

where $c>0$ and $t \in \mathbb{R} \backslash\{0\}$ are fixed constants, so that $a_{k}>0$ and $b_{k} \in \mathbb{R}$. The operator is densely defined and symmetric. Split the operator $L$ into two Jacobi matrices $J_{+}$and $J_{-}$; see [19]. The coefficients $a_{k}$ are bounded as $k \rightarrow-\infty$, so that the moment problem corresponding to $J_{-}$is determined. So the deficiency indices for $L$ are either $(0,0)$ or $(1,1)$. Take $x \in \mathbb{C} \backslash \mathbb{R}$. From the theory of orthogonal polynomials, we see that the space of solutions of $L u=x u$ which are $\ell^{2}$ for $k \rightarrow-\infty$ is one-dimensional. For the space of solutions of $L u=x u$ which are $\ell^{2}$ for $k \rightarrow \infty$, there are two possibilities: (i) the space is one-dimensional if the moment problem for $J_{+}$is determined and, in that case, $L$ is self-adjoint, and (ii) the space is twodimensional if the moment problem for $J_{+}$is indeterminate and, in that case, $L$ has a one-parameter family of self-adjoint extensions. See Akhiezer [1], Dunford and Schwartz [6, Chapter XII], and Masson and Repka [19] for more information.

Note that, for $k \rightarrow \infty$,

$$
a_{k} \pm b_{k}+a_{k-1}=c^{-\frac{1}{2}} q^{-k}\left(q^{\frac{1}{2}}+q^{-\frac{1}{2}} \pm\left(t+t^{-1}\right)\right)+q^{-\frac{1}{2}} \sqrt{c}+\mathcal{O}\left(q^{k / 2}\right)
$$

is bounded from above if $\mp t \geq q^{-\frac{1}{2}}$ or $0<\mp t \leq q^{\frac{1}{2}}$. Hence, we can use the criterion in $\left[2\right.$, Chapter VII] to see that $J_{+}$corresponds to a determined moment problem, and hence $L$ is self-adjoint, if $|t| \geq q^{-\frac{1}{2}}$ or $0<|t| \leq q^{\frac{1}{2}}$. 
Remark. The motivation to consider the operator $L$ comes from an investigation in the Hopf $*$-algebra related to the quantum $S U(1,1)$ group. This is a *-algebra with two generators $\alpha$ and $\gamma$ such that for $0<q<1$, the following relations hold:

$$
\alpha \gamma=q \gamma \alpha, \quad \alpha \gamma^{*}=q \gamma^{*} \alpha, \quad \gamma \gamma^{*}=\gamma^{*} \gamma, \quad \alpha \alpha^{*}-q^{2} \gamma \gamma^{*}=1=\alpha^{*} \alpha-\gamma^{*} \gamma .
$$

We can represent this $*$-algebra in terms of unbounded operators in $\ell^{2}(\mathbb{Z})$ by

$$
\pi(\gamma) e_{k}=\lambda q^{k} e_{k}, \quad \pi(\alpha) e_{k}=\sqrt{1+|\lambda|^{2} q^{2 k}} e_{k-1},
$$

for the standard orthonormal basis $\left\{e_{k}\right\}_{k \in \mathbb{Z}}$ of $\ell^{2}(\mathbb{Z})$ and where $\lambda \in \mathbb{C} \backslash\{0\}$. Study of the self-adjoint element $\rho=\alpha^{*} \gamma^{*}+\gamma \alpha+\left(t+t^{-1}\right) \gamma \gamma^{*}$ in these representations leads to the operator in (2.1) after some normalization. Then we might expect that the spectral resolution of $L$ will give us information on how to construct a possible Haar functional on the subalgebra generated by $\rho$. For the quantum $S U(2)$ group, this is completely rigorous, $\mathrm{cf}$. $[16, \S 5]$, and we may consider $(2.1)$ as a non-terminating version of the three-term recurrence relation for the orthonormal Al-Salam and Carlitz polynomials.

For $x \in \mathbb{C} \backslash\{0\}$, define

$$
\begin{gathered}
V_{k}^{t}(x)=\sqrt{\left(-q^{1-k} / c ; q\right)_{\infty}} q^{\frac{1}{4} k(k+1)}(-t \sqrt{c})^{k}\left(q t^{2} ; q\right)_{\infty} 1 \varphi_{1}\left(\begin{array}{c}
-t \sqrt{c} / x \\
q t^{2}
\end{array} ; q, x t q^{k+1} \sqrt{c}\right), \\
U_{k}(x)=\sqrt{\left(-q^{1-k} / c ; q\right)_{\infty}} q^{\frac{1}{4} k(k+1)} x^{k}{ }_{2} \varphi_{1}\left(\begin{array}{c}
-\sqrt{c} /(t x),-\sqrt{c} t / x \\
0
\end{array} ;,-\frac{q^{1-k}}{c}\right) .
\end{gathered}
$$

By $[7,(4.3 .2)]$, we have for $\pm t \notin\left\{q^{\frac{1}{2} m} \mid m \in \mathbb{Z}\right\}$ :

$$
\begin{gathered}
U_{k}(x)=C_{t} c_{t}(x) V_{k}^{t}(x)+C_{t^{-1}} c_{t^{-1}}(x) V_{k}^{t^{-1}}(x), \\
c_{t}(x)=(-\sqrt{c} / x t, q t / x \sqrt{c}, x \sqrt{c} / t ; q)_{\infty}, \quad C_{t}^{-1}=\left(q t^{2}, t^{-2},-c,-q / c ; q\right)_{\infty} .
\end{gathered}
$$

In the case of $\pm t \in\left\{q^{\frac{1}{2} m} \mid m \in \mathbb{Z}\right\}$, we use the fact that for $p \in \mathbb{Z}$, we have

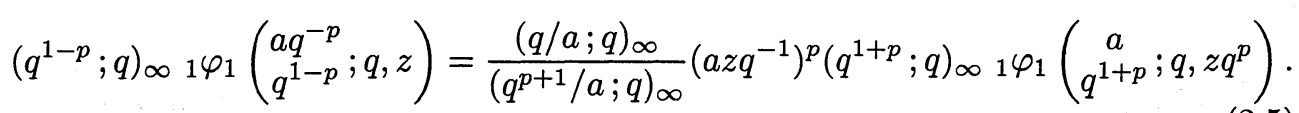

Formula (2.5) is meaningful and can be proved by interpreting its left-hand side for $p \in \mathbb{Z}_{>0}$ as

$$
\sum_{k=0}^{\infty} \frac{\left(a q^{-p} ; q\right)_{k}\left(q^{1-p+k} ; q\right)_{\infty} q^{\frac{1}{2} k(k-1)}(-z)^{k}}{(q ; q)_{k}}
$$

From (2.5), we obtain that

$$
V_{k}^{ \pm q^{-\frac{1}{2} m}}(x)=(-c)^{m} \frac{\left(\mp q^{1-\frac{1}{2} m} x / \sqrt{c} ; q\right)_{\infty}}{\left(\mp q^{1+\frac{1}{2} m} x / \sqrt{c} ; q\right)_{\infty}} V_{k}^{ \pm q^{\frac{1}{2} m}}(x)
$$

Lemma 2.1. If $x \in \mathbb{C} \backslash\{0\}$, then $V^{t}(x)=\left(V_{k}^{t}(x)\right)_{k \in \mathbb{Z}}, V^{t^{-1}}(x)=\left(V_{k}^{t^{-1}}(x)\right)_{k \in \mathbb{Z}}$, and $U(x)=\left(U_{k}(x)\right)_{k \in \mathbb{Z}}$ are solutions to Lu $=x u$. Furthermore, $V^{t}(x)$ is $\ell^{2}$ as $k \rightarrow \infty$ if and only if $|t|<q^{-\frac{1}{2}}$ or $t= \pm q^{-\frac{1}{2} m}, m \in \mathbb{Z}_{\geq 0}$, and $V^{t^{-1}}(x)$ is $\ell^{2}$ as $k \rightarrow \infty$ if and only if $|t|>q^{\frac{1}{2}}$ or $t= \pm q^{\frac{1}{2} m}, m \in \mathbb{Z}_{\geq 0}$, and $U(x)$ is $\ell^{2}$ as $k \rightarrow-\infty$ for all $t$. If, 
moreover, we assume $t \in \mathbb{R} \backslash\left\{q^{\frac{1}{2} m} \mid m \in \mathbb{Z}\right\}$, then $U(x)$ is $\ell^{2}$ as $k \rightarrow \infty$ if and only if $q^{\frac{1}{2}}<|t|<q^{-\frac{1}{2}}$.

Proof. Recall the second order $q$-difference equation

$$
(c-a b z) f(q z)+(-(c+q)+(a+b) z) f(z)+(q-z) f(z / q)=0
$$

satisfied by $f(z)={ }_{2} \varphi_{1}(a, b ; c ; q, z)$; see [7, Exercise 1.13]. Take $c=0$ to see that $U(x)$ satisfies $L u=x u$. It is clear from (2.3) that $U(x)$ is $\ell^{2}$ as $k \rightarrow-\infty$. By the confluent limit, $f(z)={ }_{1} \varphi_{1}(a ; c ; q, z)$ satisfies

$$
(c-a z) f(q z)+(-(c+q)+z) f(z)+q f(z / q)=0 .
$$

This yields that $V^{t^{ \pm 1}}(x)$ satisfies $L u=x u$. Now use the theta-product identity

$$
\left(a q^{k}, q^{1-k} / a ; q\right)_{\infty}=(-a)^{-k} q^{-\frac{1}{2} k(k-1)}(a, q / a ; q)_{\infty}, \quad \forall k \in \mathbb{Z}, a \in \mathbb{C} \backslash\{0\},
$$

to derive the statements on the $\ell^{2}$-behavior as $k \rightarrow \infty$ of $V^{t^{ \pm 1}}(x)$ together with (2.6). Use (2.4) to obtain the $\ell^{2}$-behavior as $k \rightarrow \infty$ of $U(x)$.

For $x=0$, we can take the limit in $V_{k}^{t}(x)$, e.g.,

$$
V_{k}^{t}(0)=\sqrt{\left(-q^{1-k} / c ; q\right)_{\infty}} q^{\frac{1}{4} k(k+1)}(-t \sqrt{c})^{k}\left(q t^{2} ; q\right)_{\infty} 0 \varphi_{1}\left(-; q t^{2} ; q,-q^{k+1} c t^{2}\right),
$$

which is closely related to Jackson's $q$-Bessel function $J_{\alpha}^{(2)}\left(2 \sqrt{c} q^{\frac{1}{2} k} ; q\right)$ for $t^{2}=q^{\alpha}$; see Ismail [8], and also [5, 21]. Explicitly,

$$
V_{k}^{q^{\frac{1}{2} \alpha}}(0)=\sqrt{\left(-q^{1-k} / c ; q\right)_{\infty}} q^{\frac{1}{4} k(k+1)}(-1)^{k} c^{\frac{1}{2}(k-\alpha)}(q ; q)_{\infty} J_{\alpha}^{(2)}\left(2 \sqrt{c} q^{\frac{1}{2} k} ; q\right)
$$

and a similar expression for $V_{k}^{q^{-\frac{1}{2} \alpha}}(0)$. We recall the definition of Jackson's $q$-Bessel function; see [8]:

$$
J_{\alpha}^{(2)}(x ; q):=\frac{\left(q^{\alpha+1} ; q\right)_{\infty}}{(q ; q)_{\infty}}\left(\frac{x}{2}\right)^{\alpha}{ }_{0} \varphi_{1}\left(\bar{q}_{q^{\alpha+1}}^{-} ; q,-q^{\alpha+1} \frac{x^{2}}{4}\right) .
$$

Remark. It follows from Lemma 2.1 that $L$ has deficiency indices $(1,1)$ for $q^{\frac{1}{2}}<|t|<q^{-\frac{1}{2}}, t \neq \pm 1$, and that the deficiency space $N_{ \pm i}=\left\{u \in \ell^{2}(\mathbb{Z}) \mid L u= \pm i u\right\}$ is spanned by $U( \pm i)$.

The Wronskian for two sequences $\left(u_{k}\right)_{k \in \mathbb{Z}}$ and $\left(v_{k}\right)_{k \in \mathbb{Z}}$ is given by

$$
[u, v]_{k}:=a_{k}\left(u_{k+1} v_{k}-u_{k} v_{k+1}\right) .
$$

It is independent of $k$ if $u$ and $v$ are two solutions to $L u=x u$, so that we may denote it by $[u, v]$.

Lemma 2.2. We have the following Wronskians:

$$
\begin{aligned}
{\left[V^{t}(x), V^{t^{-1}}(x)\right] } & =\frac{\sqrt{c}}{t}\left(t^{2}, q t^{-2}-1 / c,-c q ; q\right)_{\infty}, \\
{\left[U(x), V^{t}(x)\right] } & =\frac{c_{t^{-1}}(x)}{-t \sqrt{c}} .
\end{aligned}
$$

Proof. The first Wronskian follows by combining (2.10) with (2.2) and letting $k \rightarrow$ $\infty$ using (2.7). The second Wronskian follows from the first and (2.4) for $\pm t \notin$ $\left\{q^{\frac{1}{2} m} \mid m \in \mathbb{Z}\right\}$. Next use analytic continuation with respect to $t$. 
We find that for $x \in \mathbb{C} \backslash \mathbb{R}$ the solution $U(x)$ and $V^{t^{-1}}(x)$ of $L u=x u$ are linearly independent for $x \in \mathbb{C} \backslash \mathbb{R}$. Moreover, $U(x)$ is $\ell^{2}$ for $k \rightarrow-\infty$, and this determines $U(x)$ up to a constant by the considerations in the first paragraph of this section. Furthermore, $V^{t^{-1}}(x)$ is $\ell^{2}$ for $k \rightarrow \infty$ for $|t|>q^{\frac{1}{2}}$, and for $|t| \geq q^{-\frac{1}{2}}$, this condition determines $V^{t^{-1}}(x)$ up to a constant.

\section{Spectral resolution}

In case $|t|>q^{\frac{1}{2}}$, we see $V^{t^{-1}}(x)$ is $\ell^{2}$ for $k \rightarrow \infty$ by Lemma 2.1. From now on we assume that $|t|>q^{\frac{1}{2}}$. In case $|t| \geq q^{-\frac{1}{2}}$, we also have that $L$ is self-adjoint. The domain of $L$ is given by $\left\{u \in \ell^{2}(\mathbb{Z}) \mid L u \in \ell^{2}(\mathbb{Z})\right\}$. In case $q^{\frac{1}{2}}<|t|<q^{-\frac{1}{2}}, t \neq \pm 1$, the deficiency indices of $L$ are $(1,1)$ so that all extensions of $L$ to a self-adjoint operator are parametrized by $U(1)=\mathbb{T}$. In this case, we fix the self-adjoint extension such that $V^{t^{-1}}$ is contained in the domain, which is always possible since $\overline{N_{i}}=N_{-i}$ because of $a_{k}, b_{k} \in \mathbb{R}$. See Dunford and Schwartz [6, Chapter XII] for more information.

Define the Green's function for $x \in \mathbb{C} \backslash \mathbb{R}$ by

$$
G_{x}(m, n):= \begin{cases}\frac{U_{n}(x) V_{m}^{t^{-1}}(x)}{\left[U(x), V^{t^{-1}}(x)\right]}, & \text { for } n \leq m, \\ \frac{U_{m}(x) V_{n}^{t^{-1}}(x)}{\left[U(x), V^{t^{-1}}(x)\right]}, & \text { for } n>m,\end{cases}
$$

so that $\left((x-L)^{-1} f\right)_{m}=\sum_{n=-\infty}^{\infty} G_{x}(m, n) f_{n}$. For the resolution of the identity $E$ for $L$, i.e., $L=\int_{\mathbb{R}} t d E(t)$, we now have (see [6, Chapter XII])

$$
\langle E((a, b)) v, w\rangle=\lim _{\delta \downarrow 0} \lim _{\varepsilon \downarrow 0} \frac{1}{2 \pi i} \int_{a+\delta}^{b-\delta}\left\langle(s-i \varepsilon-L)^{-1} v, w\right\rangle-\left\langle(s+i \varepsilon-L)^{-1} v, w\right\rangle d s
$$

Now observe that

$$
\left\langle(s \pm i \varepsilon-L)^{-1} v, w\right\rangle=\sum_{n \leq m} \frac{U_{n}(s \pm i \varepsilon) V_{m}^{t^{-1}}(s \pm i \varepsilon)}{\left[U(s \pm i \varepsilon), V^{t^{-1}}(s \pm i \varepsilon)\right]}\left(v_{n} \bar{w}_{m}+v_{m} \bar{w}_{n}\right)\left(1-\frac{1}{2} \delta_{n, m}\right)
$$

Since $V_{m}^{t^{-1}}(x)$ is an entire function of $x$, and $U_{n}(x)$ is analytic in $\mathbb{C} \backslash\{0\}$, we see that the measure $\langle E((a, b)) v, w\rangle$ has only discrete mass points at the zeros of the Wronskian $\left[U(x), V^{t^{-1}}(x)\right]$. By Lemma 2.2, this corresponds to the zeros of $c_{t}(x)$ which are the points $\eta_{p}=-\sqrt{c} q^{p} / t, p \in \mathbb{Z}_{\geq 0}$, and $\xi_{p}=t q^{p} / \sqrt{c}, p \in \mathbb{Z}$. So the spectrum of $L$ is $\left\{\eta_{p} \mid p \in \mathbb{Z}_{\geq 0}\right\} \cup\left\{\xi_{p} \mid p \in \mathbb{Z}\right\} \cup\{0\}$, and it remains to show that 0 is not in the point spectrum of $L$. It follows from Moak [21, §7] and (2.8) that $V_{k}^{t^{-1}}(0)$ is not identically zero for $k<-N$ for some $N$. The observation now follows from the asymptotic expression for the Jackson $q$-Bessel function derived by Chen et al. [5, Theorem 4], which shows that the Jackson $q$-Bessel function in (2.8) increases exponentially with $k^{2}$ as $k \rightarrow-\infty$.

In order to calculate $E\left(\left\{\eta_{p}\right\}\right)$, we take the interval $(a, b)$ such that it contains only $\eta_{p}$ as a point from the spectrum. Then 


$$
\begin{aligned}
& \left\langle E\left(\left\{\eta_{p}\right\}\right) v, w\right\rangle=\langle E((a, b)) v, w\rangle \\
& =\lim _{\varepsilon \downarrow 0} \frac{1}{2 \pi i} \int_{a}^{b}\left\langle(s-i \varepsilon-L)^{-1} v, w\right\rangle-\left\langle(s+i \varepsilon-L)^{-1} v, w\right\rangle d s \\
& =\frac{1}{2 \pi i} \oint_{\left(\eta_{p}\right)}\left\langle(s-L)^{-1} v, w\right\rangle d s \\
& =\sum_{n \leq m}\left(v_{n} \bar{w}_{m}+v_{m} \bar{w}_{n}\right)\left(1-\frac{1}{2} \delta_{n, m}\right) \frac{1}{2 \pi i} \oint_{\left(\eta_{p}\right)} \frac{U_{n}(s) V_{m}^{t^{-1}}(s)}{\left[U(s), V^{t^{-1}}(s)\right]} d s \\
& =\sum_{n \leq m}\left(v_{n} \bar{w}_{m}+v_{m} \bar{w}_{n}\right)\left(1-\frac{1}{2} \delta_{n, m}\right) U_{n}\left(\eta_{p}\right) V_{m}^{t^{-1}}\left(\eta_{p}\right)\left(-\sqrt{c} t^{-1}\right) \underset{x=\eta_{p}}{\operatorname{Res}} \frac{1}{c_{t}(x)} \\
& =C_{t^{-1}} c_{t^{-1}}\left(\eta_{p}\right)\left(-\sqrt{c} t^{-1}\right) \operatorname{Res}_{x=\eta_{p}} \frac{1}{c_{t}(x)}\left\langle v, V^{t^{-1}}\left(\eta_{p}\right)\right\rangle\left\langle V^{t^{-1}}\left(\eta_{p}\right), w\right\rangle
\end{aligned}
$$

by (2.4) and $c_{t}\left(\eta_{p}\right)=0$. Since ${ }_{1} \varphi_{1}\left(q^{-p} ; q t^{-2} ; q,-c q^{p+k+1} t^{-2}\right) \sim C q^{k p}$ for $k \rightarrow-\infty$, we obtain $V^{t^{-1}}\left(\eta_{p}\right) \in \ell^{2}(\mathbb{Z})$ also in a direct manner. Next

$$
\operatorname{Res}_{x=\eta_{p}} \frac{1}{c_{t}(x)}=\frac{1}{\left(-q^{1-p} t^{2} / c,-q^{p} c / t^{2} ; q\right)_{\infty}} \frac{-q^{p} \sqrt{c} / t}{\left(q^{-p} ; q\right)_{p}(q ; q)_{\infty}}
$$

and

$$
C_{t^{-1}} c_{t^{-1}}\left(\eta_{p}\right)=\frac{\left(t^{2} q^{-p} ; q\right)_{p}}{\left(q t^{-2} ; q\right)_{\infty}} c^{-p} q^{-\frac{1}{2} p(p-1)}
$$

by (2.7). So that finally,

$$
\begin{aligned}
& \left\langle E\left(\left\{\eta_{p}\right\}\right) v, w\right\rangle \\
& \quad=c t^{-2} q^{p} \frac{\left(q t^{-2} ; q\right)_{p}}{(q ; q)_{p}} \frac{1}{\left(-c / t^{2},-q t^{2} / c, q t^{-2} ; q\right)_{\infty}}\left\langle v, V^{t^{-1}}\left(\eta_{p}\right)\right\rangle\left\langle V^{t^{-1}}\left(\eta_{p}\right), w\right\rangle .
\end{aligned}
$$

Take $v=w=V^{t^{-1}}\left(\eta_{p}\right)$ and use that $E\left(\left\{\eta_{p}\right\}\right) V^{t^{-1}}\left(\eta_{p}\right)=V^{t^{-1}}\left(\eta_{p}\right)$ to see that

$$
\left\|V^{t^{-1}}\left(\eta_{p}\right)\right\|^{2}=c^{-1} t^{2} q^{-p} \frac{(q ; q)_{p}}{\left(q t^{-2} ; q\right)_{p}}\left(q,-c / t^{2},-q t^{2} / c, q t^{-2} ; q\right)_{\infty} .
$$

Since the projections $E\left(\left\{\eta_{p}\right\}\right)$ satisfy $E\left(\left\{\eta_{p}\right\}\right) E\left(\left\{\eta_{r}\right\}\right)=\delta_{p r} E\left(\left\{\eta_{p}\right\}\right)$, we get

$$
\begin{aligned}
&\left\langle V^{t^{-1}}\left(\eta_{p}\right),\right.\left.V^{t^{-1}}\left(\eta_{r}\right)\right\rangle=\left\langle E\left(\left\{\eta_{p}\right\}\right) V^{t^{-1}}\left(\eta_{p}\right), E\left(\left\{\eta_{r}\right\}\right) V^{t^{-1}}\left(\eta_{r}\right)\right\rangle \\
&=\left\langle V^{t^{-1}}\left(\eta_{p}\right), E\left(\left\{\eta_{p}\right\}\right) E\left(\left\{\eta_{r}\right\}\right) V^{t^{-1}}\left(\eta_{r}\right)\right\rangle=\delta_{p r}\left\langle V^{t^{-1}}\left(\eta_{p}\right), V^{t^{-1}}\left(\eta_{p}\right)\right\rangle
\end{aligned}
$$

and, after setting $t^{-2}=q^{\alpha}, \alpha>-1$, we obtain from (3.2) and (3.3) the orthogonality relations for the $q$-Laguerre polynomials on the set $\left\{c q^{k} \mid k \in \mathbb{Z}\right\}, c>0$, obtained by Moak [21]. It is well known that these polynomials correspond to an indeterminate moment problem, and that the orthogonality measure supported on $\left\{c q^{k} \mid k \in \mathbb{Z}\right\}$ is not $\mathrm{N}$-extremal, i.e., the polynomials are not dense in the corresponding $L^{2}$-space; see $[1,21]$.

Similarly,

$$
\left\langle E\left(\left\{\xi_{p}\right\}\right) v, w\right\rangle=C_{t^{-1}} c_{t^{-1}}\left(\xi_{p}\right)\left(-\sqrt{c} t^{-1}\right) \operatorname{Res}_{x=\xi_{p}} \frac{1}{c_{t}(x)}\left\langle v, V^{t^{-1}}\left(\xi_{p}\right)\right\rangle\left\langle V^{t^{-1}}\left(\xi_{p}\right), w\right\rangle
$$


with

$$
\begin{aligned}
\operatorname{Res}_{x=\xi_{p}} \frac{1}{c_{t}(x)} & =\frac{t q^{p}}{\sqrt{c}} \frac{(-1)^{p-1} q^{\frac{1}{2} p(p-1)}}{\left(-c q^{-p} t^{-2}, q, q ; q\right)_{\infty}}, \\
C_{t^{-1}} c_{t^{-1}}\left(\xi_{p}\right) & =\left(-\frac{c}{t^{2}}\right)^{p} q^{-p^{2}} \frac{1}{\left(-q^{1+p} / c ; q\right)_{\infty}} .
\end{aligned}
$$

Hence,

$$
\left\langle E\left(\left\{\xi_{p}\right\}\right) v, w\right\rangle=q^{p} \frac{\left(-q^{p+1} t^{2} / c ; q\right)_{\infty}}{\left(-q^{p+1} / c, q, q,-c t^{-2},-q t^{2} / c ; q\right)_{\infty}}\left\langle v, V^{t^{-1}}\left(\xi_{p}\right)\right\rangle\left\langle V^{t^{-1}}\left(\xi_{p}\right), w\right\rangle .
$$

Similarly, as before, we obtain

$$
\left\langle V^{t^{-1}}\left(\xi_{p}\right), V^{t^{-1}}\left(\xi_{r}\right)\right\rangle=\delta_{p r} q^{-p} \frac{\left(-q^{p+1} / c, q, q,-c t^{-2},-q t^{2} / c ; q\right)_{\infty}}{\left(-q^{p+1} t^{2} / c ; q\right)_{\infty}} .
$$

Remark. The vector $V^{t^{-1}}\left(\xi_{p}\right)$ belongs to $\ell^{2}(\mathbb{Z})$ for $p \in \mathbb{Z}$. This also can be seen as follows. First, apply to (2.3) the transformation formula

$$
{ }_{1} \varphi_{1}\left(\begin{array}{l}
a \\
c
\end{array} ; q, z\right)=\frac{(z ; q)_{\infty}}{(c ; q)_{\infty}}{ }_{1} \varphi_{1}\left(\begin{array}{c}
a z / c \\
z
\end{array} ; q, c\right)
$$

(a limit case of Heine's transformation formula [7, (1.4.5)]). The combination of (2.3), (3.5), and (2.5) gives

$$
\begin{aligned}
& V_{k}^{t^{-1}}\left(\xi_{p}\right)=\frac{\left(-q^{p+1} / c ; q\right)_{\infty}\left(-t^{2} c^{-1} q^{p}\right)^{p}}{\sqrt{\left(-q^{1-k} / c ; q\right)_{\infty}}\left(q t^{-2} ; q\right)_{\infty}} q^{\frac{1}{4} k(k+1)}\left(t c^{-\frac{1}{2}} q^{p}\right)^{k}\left(q t^{-2} ; q\right)_{\infty} \\
& \times\left(q^{1-p-k} ; q\right)_{\infty 1} \varphi_{1}\left(\begin{array}{c}
-c q^{-p} \\
q^{1-p-k} ; q, q^{1-p-k} t^{-2}
\end{array}\right),
\end{aligned}
$$

so that we obtain the $\ell^{2}$-behavior as $k \rightarrow-\infty$ of $V^{t^{-1}}(x)$.

\section{Basis for $L^{2}$-space}

By $L^{2}\left(\mu^{(\alpha ; c)}\right)$, we denote the space of square integrable functions on the set $\left\{c q^{k} \mid k \in\right.$ $\mathbb{Z}\}, c>0$, with positive weight $q^{k(\alpha+1)} /\left(-c q^{k} ; q\right)_{\infty}$ at $c q^{k}, k \in \mathbb{Z}$, i.e., $f \in L^{2}\left(\mu^{(\alpha ; c)}\right)$ if

$$
\mathcal{L}\left(|f|^{2}\right):=\sum_{k=-\infty}^{\infty} \frac{q^{k(\alpha+1)}}{\left(-c q^{k} ; q\right)_{\infty}}\left|f\left(c q^{k}\right)\right|^{2}<\infty
$$

Here we take $\alpha>-1$.

Recall the $q$-Laguerre polynomials introduced by Moak [21] (see also [7])

$$
L_{n}^{(\alpha)}(x ; q):=\frac{\left(q^{\alpha+1} ; q\right)_{n}}{(q ; q)_{n}}{ }_{1} \varphi_{1}\left(\begin{array}{c}
q^{-n} \\
q^{\alpha+1}
\end{array} ;,-x q^{n+\alpha+1}\right) .
$$

We also define the functions

$$
\begin{aligned}
M_{p}^{(\alpha ; c)}(x ; q) & :=\frac{\left(q^{\alpha+1} ; q\right)_{\infty}}{\left(q,-c q^{\alpha+1} ; q\right)_{\infty}}{ }_{1} \varphi_{1}\left(\begin{array}{c}
-c q^{\alpha-p} \\
q^{\alpha+1} ; q, \frac{x q^{p+1}}{c}
\end{array}\right) \\
& =\frac{\left(x q^{p+1} / c ; q\right)_{\infty}}{\left(q,-c q^{\alpha+1} ; q\right)_{\infty}}{ }_{1} \varphi_{1}\left(\begin{array}{c}
-x \\
x q^{p+1} / c
\end{array} ;, q^{\alpha+1}\right)
\end{aligned}
$$

for $p \in \mathbb{Z}$. (The second equality is by (3.5).) 
Theorem 4.1. The functions $M_{p}^{(\alpha ; c)}(\cdot ; q), p \in \mathbb{Z}$, together with the $q$-Laguerre polynomials $L_{n}^{(\alpha)}(\cdot ; q), n \in \mathbb{Z}_{\geq 0}$, form an orthogonal basis for $L^{2}\left(\mu^{(\alpha ; c)}\right), c>0, \alpha>-1$. Explicitly,

$$
\begin{aligned}
\mathcal{L}\left(L_{n}^{(\alpha)}(\cdot ; q) L_{p}^{(\alpha)}(\cdot ; q)\right) & =\delta_{n, p} q^{-p} \frac{\left(q^{\alpha+1} ; q\right)_{p}}{(q ; q)_{p}} \frac{\left(q,-c q^{\alpha+1},-q^{-\alpha} / c ; q\right)_{\infty}}{\left(q^{\alpha+1},-c,-q / c ; q\right)_{\infty}} \\
\mathcal{L}\left(M_{p}^{(\alpha ; c)}(\cdot ; q) M_{r}^{(\alpha ; c)}(\cdot ; q)\right) & =\delta_{p, r} c q^{\alpha} q^{-p} \frac{\left(-q^{p+1} / c,-q^{-\alpha} / c ; q\right)_{\infty}}{\left(-q^{p+1-\alpha} / c,-c q^{\alpha+1} ; q\right)_{\infty}} \frac{1}{(-c,-q / c ; q)_{\infty}} \\
\mathcal{L}\left(M_{p}^{(\alpha ; c)}(\cdot ; q) L_{n}^{(\alpha)}(\cdot ; q)\right) & =0 .
\end{aligned}
$$

Note that $M_{p}^{(\alpha ; c)}(x ; q)$ depends on $c$, unlike $L_{n}^{(\alpha)}(x ; q)$. It also should be observed that $M_{p}^{(\alpha ; c)}\left(c q^{k} ; q\right)$ is bounded as $k \rightarrow \infty$ and that $M_{p}^{(\alpha ; c)}\left(c q^{k} ; q\right) \rightarrow 0$ for $\left|q^{p-\alpha} / c\right|<1$ for $k \rightarrow-\infty$ as follows from (2.5). Using the method of Berg [3], we can construct more orthogonality measures for the $q$-Laguerre polynomials supported on the set $\left\{c q^{k} \mid k \in \mathbb{Z}\right\}$. Let then $\left|M_{p}^{(\alpha ; c)}\left(c q^{k} ; q\right)\right| \leq K$ for some $K$ for any $p \in \mathbb{Z}$ satisfying $\left|q^{p-\alpha} / c\right|<1$. Then the $q$-Laguerre polynomials are also orthogonal with respect to the positive discrete measure with masses $\left(1+s K^{-1} M_{p}^{(\alpha ; c)}\left(c q^{k} ; q\right)\right) q^{k(\alpha+1)} /\left(-c q^{k} ; q\right)_{\infty}$ at $c q^{k}, k \in \mathbb{Z}$, for any $s \in[-1,1]$; cf. [3, Proposition 4.1]. We also may take suitable linear combinations of the functions $M_{p}^{(\alpha ; c)}(x ; q)$.

Proof. Apply the results of the previous section with $t^{-2}=q^{\alpha}$. The first statement corresponds to $\left\langle V^{t^{-1}}\left(\eta_{p}\right), V^{t^{-1}}\left(\eta_{r}\right)\right\rangle=\delta_{p, r}\left\|V^{t^{-1}}\left(\eta_{p}\right)\right\|^{2}$, the second statement corresponds to $\left\langle V^{t^{-1}}\left(\xi_{p}\right), V^{t^{-1}}\left(\xi_{r}\right)\right\rangle=\delta_{p, r}\left\|V^{t^{-1}}\left(\xi_{p}\right)\right\|^{2}$, and the last one to $\left\langle V^{t^{-1}}\left(\xi_{p}\right), V^{t^{-1}}\right.$ $\left.\left(\eta_{r}\right)\right\rangle=0$.

Remark. The first statement of Theorem 4.1 corresponds to Moak's discrete orthogonality relations [21, Theorem 2] for the $q$-Laguerre polynomials. The second relation can be rewritten as

$$
\begin{aligned}
\sum_{k=-\infty}^{\infty} \frac{q^{k(\alpha+1)}\left(q^{\alpha+1} ; q\right)_{\infty}}{\left(-c q^{k} ; q\right)_{\infty}(q ; q)_{\infty}} 1 \varphi_{1}\left(\begin{array}{c}
-c q^{\alpha-p} \\
\left.q^{\alpha+1} ; q, q^{p+k+1}\right)
\end{array}\right. \\
\quad \times \frac{\left(q^{\alpha+1} ; q\right)_{\infty}}{(q ; q)_{\infty}} 1_{1} \varphi_{1}\left(\begin{array}{c}
-c q^{\alpha-r} \\
\left.q^{\alpha+1} ; q, q^{r+k+1}\right)
\end{array}\right. \\
=\delta_{p, r} c q^{\alpha} q^{-p} \frac{\left(-q^{p+1} / c ; q\right)_{\infty}}{\left(-q^{p+1-\alpha} / c ; q\right)_{\infty}} \frac{\left(-c q^{\alpha+1},-q^{-\alpha} / c ; q\right)_{\infty}}{(-c,-q / c ; q)_{\infty}} \\
=\sum_{k=-\infty}^{\infty} \frac{q^{k(\alpha+1)}\left(q^{k+p+1} ; q\right)_{\infty}}{\left(-c q^{k} ; q\right)_{\infty}(q ; q)_{\infty}} \varphi_{1}\left(\begin{array}{c}
-c q^{k} \\
q^{k+p+1} ; q, q^{\alpha+1}
\end{array}\right) \\
\quad \times \frac{\left(q^{k+r+1} ; q\right)_{\infty}}{(q ; q)_{\infty}} 1 \varphi_{1}\left(\begin{array}{c}
-c q^{k} \\
q^{k+r+1}
\end{array} ;, q^{\alpha+1}\right)
\end{aligned}
$$

for $p, r \in \mathbb{Z}$, which can be viewed as a $q$-analogue of the Hankel transform for the 
first equality or as a $q$-analogue of the Hansen-Lommel orthogonality relations for the second equality, cf. [18]. The limit case $c \rightarrow 0$ corresponds to [18, Proposition 2.6]. The last statement shows that these $q$-Bessel functions are orthogonal to the $q$-Laguerre polynomials;

$$
\begin{aligned}
& \sum_{k=-\infty}^{\infty} \frac{q^{k(\alpha+1)}}{\left(-c q^{k} ; q\right)_{\infty}} L_{n}^{(\alpha)}\left(c q^{k} ; q\right) \frac{\left(q^{\alpha+1} ; q\right)_{\infty}}{(q ; q)_{\infty}} 1 \varphi_{1}\left(\begin{array}{c}
-c q^{\alpha-r} \\
\left.q^{\alpha+1} ; q, q^{r+k+1}\right)
\end{array}\right)=0 \\
& r \in \mathbb{Z}, n \in \mathbb{Z}_{\geq 0}
\end{aligned}
$$

Since the statement is that $\left\{V^{t^{-1}}\left(\eta_{p}\right)\right\}_{p \in \mathbb{Z} \geq 0}$ and $\left\{V^{t^{-1}}\left(\xi_{p}\right)\right\}_{p \in \mathbb{Z}}$ form an orthogonal basis for the Hilbert space $\ell^{2}(\mathbb{Z})$, we also find the dual orthogonality relations

$$
\sum_{p=0}^{\infty} \frac{V_{k}^{t^{-1}}\left(\eta_{p}\right) V_{l}^{t^{-1}}\left(\eta_{p}\right)}{\left\|V^{t^{-1}}\left(\eta_{p}\right)\right\|^{2}}+\sum_{p=-\infty}^{\infty} \frac{V_{k}^{t^{-1}}\left(\xi_{p}\right) V_{l}^{t^{-1}}\left(\xi_{p}\right)}{\left\|V^{t^{-1}}\left(\xi_{p}\right)\right\|^{2}}=\delta_{k, l}
$$

The first sum is the Poisson kernel for the $q$-Laguerre polynomials evaluated at one; it also can be derived from the Christoffel-Darboux formula and the limit transition of the $q$-Laguerre polynomials to Jackson's $q$-Bessel function; see [21]. Explicitly, from $[21,(4.11)$, Theorem 5], we get

$$
\begin{aligned}
& \sum_{p=0}^{N} \frac{q^{p}(q ; q)_{p}}{\left(q^{\alpha+1} ; q\right)_{p}} L_{p}^{(\alpha)}(x ; q) L_{p}^{(\alpha)}(y ; q) \\
& \quad=\frac{(q ; q)_{N}}{\left(q^{\alpha+1} ; q\right)_{N}} \frac{1}{x-y}\left(x L_{N}^{(\alpha+1)}(x ; q) L_{N}^{(\alpha)}(y ; q)-y L_{N}^{(\alpha+1)}(y ; q) L_{N}^{(\alpha)}(x ; q)\right) \stackrel{N \rightarrow \infty}{\longrightarrow} \\
& \frac{(q ; q)_{\infty}}{\left(q^{\alpha+1} ; q\right)_{\infty}} \frac{(x y)^{-\frac{1}{2} \alpha}}{x-y}\left(\sqrt{x} J_{\alpha+1}^{(2)}(2 \sqrt{x} ; q) J_{\alpha}^{(2)}(2 \sqrt{y} ; q)-\sqrt{y} J_{\alpha+1}^{(2)}(2 \sqrt{y} ; q) J_{\alpha}^{(2)}(2 \sqrt{x} ; q)\right)
\end{aligned}
$$

where the right-hand side is well defined for $x=y$ using l'Hôpital's formula. Using $t^{-2}=q^{\alpha}$, we get

$$
\begin{gathered}
\sum_{p=0}^{\infty} \frac{V_{k}^{t^{-1}}\left(\eta_{p}\right) V_{l}^{t^{-1}}\left(\eta_{p}\right)}{\left\|V^{t^{-1}}\left(\eta_{p}\right)\right\|^{2}}=\frac{q^{\frac{1}{2}(a+1)(k+l)}(-1)^{k+l}}{\left(-c q^{k},-c q^{l} ; q\right)_{\infty}^{\frac{1}{2}}} \frac{\left(-c,-q / c, q^{\alpha+1} ; q\right)_{\infty}}{\left(-c q^{\alpha+1},-q^{-\alpha} / c, q ; q\right)_{\infty}} \\
\quad \times \sum_{p=0}^{\infty} \frac{q^{p}(q ; q)_{p}}{\left(q^{\alpha+1} ; q\right)_{p}} L_{p}^{(\alpha)}\left(c q^{k} ; q\right) L_{p}^{(\alpha)}\left(c q^{l} ; q\right) \\
=\frac{q^{\frac{1}{2}(k+l)}(-1)^{k+l} c^{-\alpha-\frac{1}{2}}(-c,-q / c ; q)_{\infty}}{\left(-c q^{k},-c q^{l} ; q\right)_{\infty}^{\frac{1}{2}}\left(-c q^{\alpha+1},-q^{-\alpha} / c ; q\right)_{\infty}} \frac{1}{q^{k}-q^{l}} \\
\quad \times\left(q^{\frac{1}{2} k} J_{\alpha+1}^{(2)}\left(2 \sqrt{c} q^{\frac{1}{2} k} ; q\right) J_{\alpha}^{(2)}\left(2 \sqrt{c} q^{\frac{1}{2} l} ; q\right)-q^{\frac{1}{2} l} J_{\alpha+1}^{(2)}\left(2 \sqrt{c} q^{\frac{1}{2} l} ; q\right) J_{\alpha}^{(2)}\left(2 \sqrt{c} q^{\frac{1}{2} k} ; q\right)\right)
\end{gathered}
$$

by (4.5). Calculating the second sum in (4.4) is straightforward, so that we now have obtained the following corollary. 
Corollary 4.2. The following orthogonality relations hold:

$$
\begin{aligned}
\delta_{k, l} c q^{-k}\left(-c q^{k} ; q\right)_{\infty} \frac{\left(-c q^{\alpha+1},-q^{-\alpha} / c ; q\right)_{\infty}}{(-c,-q / c ; q)_{\infty}} & =\frac{c^{\frac{1}{2}-\alpha}}{q^{k}-q^{l}} \\
\times\left(q^{\frac{1}{2} k} J_{\alpha+1}^{(2)}\left(2 \sqrt{c} q^{\frac{1}{2} k} ; q\right) J_{\alpha}^{(2)}\left(2 \sqrt{c} q^{\frac{1}{2} l} ; q\right)\right. & \left.-q^{\frac{1}{2} l} J_{\alpha+1}^{(2)}\left(2 \sqrt{c} q^{\frac{1}{2} l} ; q\right) J_{\alpha}^{(2)}\left(2 \sqrt{c} q^{\frac{1}{2} k} ; q\right)\right) \\
+q^{\alpha\left(\frac{1}{2}(k+l)-1\right)} \sum_{p=-\infty}^{\infty} q^{p} \frac{\left(-q^{p+1-\alpha} / c ; q\right)_{\infty}}{\left(-q^{p+1} / c ; q\right)_{\infty}} & \frac{\left(q^{\alpha+1} ; q\right)_{\infty}}{(q ; q)_{\infty}}{ }_{1} \varphi_{1}\left(\begin{array}{c}
-c q^{\alpha-p} \\
q^{\alpha+1} ; q, q^{k+p+1}
\end{array}\right) \\
\times & \times \frac{\left(q^{\alpha+1} ; q\right)_{\infty}}{(q ; q)_{\infty}} \varphi_{1}\left(\begin{array}{c}
-c q^{\alpha-p} \\
q^{\alpha+1}
\end{array} ; q, q^{l+p+1}\right) .
\end{aligned}
$$

In this form, Corollary 4.2 is reminiscent of the Hankel transform; whereas if we use the transformation for the ${ }_{1} \varphi_{1}$-series of (4.2), the orthogonality relations remind us of the Hansen-Lommel orthogonality relations; cf. [18]. Of course, we may also replace the expression for the Jackson $q$-Bessel function by the Poisson kernel for the $q$-Laguerre polynomials evaluated at one, cf. (4.5), to obtain the dual orthogonality relations involving a sum over $\mathbb{Z}$ and over $\mathbb{Z}_{\geq 0}$.

\section{Direct proof of the orthogonality relations of Theorem 4.1}

In this section, we present a direct analytic proof using summation and transformation formulas of the orthogonality relations of Theorem 4.1, but not of the completeness. The first statement of the orthogonality relations is well known, see Moak [21], so we concentrate on the last two. The method is based on manipulating generating functions, cf. $[18,13]$. We start with the following lemma giving generating functions for the $q$-Bessel functions under consideration.

Lemma 5.1. We have the following generating functions:

(i) For $0<|z|<|b|^{-1}$, we have

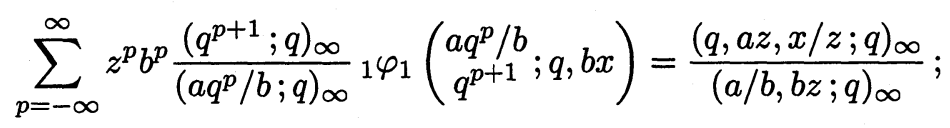

(ii) for $|d|<|w|<1$, we have

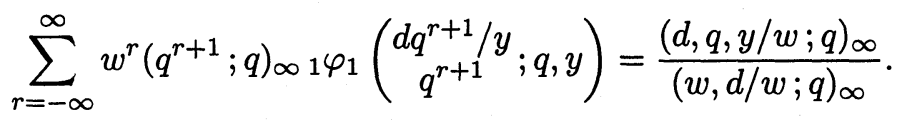

Proof. Case (i) is a limit case of [13, Proposition 2.1]. Use the $q$-binomial theorem $[7,(1.3 .2)]$ to expand $(a z ; q)_{\infty} /(b z ; q)_{\infty}$ in a power series of $z$ for $|z|<|b|^{-1}$, and $[7,(1.3 .16)]$ to expand $(x / z ; q)_{\infty}$ in a power series in $z^{-1}$ for $|z|>0$. Multiply the resulting series to find the result.

For the proof of (ii), we expand $1 /(w ; q)_{\infty}$ in a power series in $w$ for $|w|<1$, and $(y / w ; q)_{\infty} /(d / w ; q)_{\infty}$ in a power series in $w^{-1}$ for $|d|<|w|$ using [7, (1.3.2)] twice. Combine this to write the right-hand side as a Laurent series in $w$ with coefficients given by a ${ }_{2} \varphi_{1}$-series. Then use the limit case $b \rightarrow 0$ of Heine's transformation [7, (1.4.6)] to get the result. 
To prove the last part of Theorem 4.1, we use Lemma 5.1(i), after replacing $p$ by $k+r, a$ by $-b c q^{-r}$, and $x$ by $q^{\alpha+1} / b$ to get

$$
\sum_{k=-\infty}^{\infty} \frac{(z b)^{k+r}}{\left(-c q^{k} ; q\right)_{\infty}}\left(q^{k+r+1} ; q\right)_{\infty 1} \varphi_{1}\left(\begin{array}{c}
-c q^{k} \\
q^{k+r+1}
\end{array} ; q, q^{\alpha+1}\right)=\frac{\left(q,-b z c q^{-r}, q^{\alpha+1} /(b z) ; q\right)_{\infty}}{\left(-c q^{-r}, b z ; q\right)_{\infty}}
$$

Specializing $z b=q^{\alpha+1+m}, \alpha>-1$, shows that the right-hand side is zero for $m \in \mathbb{Z}_{\geq 0}$ whereas the left-hand side is a non-zero multiple of $\mathcal{L}$ applied to $M_{r}^{(\alpha ; c)}(x ; q) x^{m}$. So $M_{r}^{(\alpha ; c)}(x ; q)$ is orthogonal to all monomials, hence to all polynomials, implying the last statement of Theorem 4.1.

To prove the orthogonality relations for the functions $M^{(\alpha ; c)}(x ; q)$ of Theorem 4.1, we first deduce the result.

Proposition 5.2. For $|y|<1, l \in \mathbb{Z}$, we have

$$
\begin{gathered}
\sum_{k=-\infty}^{\infty} \frac{y^{k}}{\left(a q^{k} / b ; q\right)_{\infty}}\left(q^{k+1} ; q\right)_{\infty 1} \varphi_{1}\left(\begin{array}{c}
a q^{k} / b \\
\left.q^{k+1} ; q, y\right)\left(q^{k-l+1} ; q\right)_{\infty 1} \varphi_{1}\left(\begin{array}{c}
d q^{k-l+1} / y \\
q^{k-l+1}
\end{array} ;, y\right)
\end{array}\right. \\
\quad= \begin{cases}0, & \text { for } l<0, \\
d^{l} \frac{(a y /(b d) ; q)_{l}(d, q, q ; q)_{\infty}}{(q ; q)_{l}(a / b ; q)_{\infty}}, & \text { for } l \geq 0 .\end{cases}
\end{gathered}
$$

Proof. Choose $w=y /(b z)$ in Lemma 5.1(ii) and multiply by the generating function of Lemma $5.1(\mathrm{i})$ with $x$ replaced by $y / b$. This then gives the identity

$$
\begin{aligned}
& \frac{(a z ; q)_{\infty}(d, q, q ; q)_{\infty}}{(b z d / y ; q)_{\infty}(a / b ; q)_{\infty}}=\sum_{l=-\infty}^{\infty} z^{l} \sum_{k=-\infty}^{\infty} \frac{y^{k-l} b^{l}}{\left(a q^{k} / b ; q\right)_{\infty}} \\
& \times\left(q^{k+1} ; q\right)_{\infty 1} \varphi_{1}\left(\begin{array}{c}
a q^{k} / b \\
q^{k+1}
\end{array} ; q, y\right)\left(q^{k-l+1} ; q\right)_{\infty 1} \varphi_{1}\left(\begin{array}{c}
d q^{k-l+1} / y \\
q^{k-l+1}
\end{array} q, y\right)
\end{aligned}
$$

under the condition $|y / b|<|z|<\min \left(|b|^{-1},|y /(d b)|\right)$. We need $|y|<1,|d|<1$ to have a non-empty region of analyticity of the right-hand side as a function of $z$. The left-hand side is an analytic function of $z$ in $|z|<|y /(d b)|$, and it can be expanded in a power series in $z$ using the $q$-binomial theorem [7, (1.3.2)]. Comparing coefficients on both sides gives the result for $|d|<1$.

Next we use analytic continuation with respect to $d$. For fixed $l \in \mathbb{Z}$, the righthand side is analytic in $d$. To see that the left-hand side is analytic in $d$, we note that $f_{k}(d)=\left(q^{k-l+1} ; q\right)_{\infty 1} \varphi_{1}\left(d q^{k-l+1} / y ; q^{k-l+1} ; q, y\right)$ is analytic in $d$. Moreover, for $k \geq l$, we easily estimate $\left|f_{k}(d)\right| \leq(-|d / y|,-|y| ; q)_{\infty}$, so that the convergence of the sum for $k \rightarrow \infty$ is uniform in $d$ on compact subsets since $|y|<1$.

In order to obtain uniform convergence as $k \rightarrow-\infty$, we use (2.5) so that for $k \leq-l$, we can estimate $\left|f_{k}(d)\right| \leq\left|d^{l-k}(y / d ; q)_{l-k}\right|(-|d / y|,-|y| ; q)_{\infty}$. On the other hand, we see that by (2.5) and (2.7), the coefficient for the function $f_{k}(d)$ as $k \rightarrow \infty$ behaves like $C(-1)^{k} q^{\frac{1}{2} k(k+1)}$. Hence, the convergence for $k \rightarrow-\infty$ also is uniform in $d$ on compact subsets. Hence, both sides of the equality of this proposition are analytic in $d$ and coincide on $|d|<1$, so it holds for all $d$.

There are many ways of linking $w$ and $z$ such that the products of the generating functions of Lemma 5.1 simplify. Taking $w=x / z$ and $b x=y$ leads to the same result. 
The case $a z=d / w$ also gives an interesting result, which may be obtained as a limit case of [13, Proposition 2.2].

In Proposition 5.2 , we replace $k$ by $k+p$ and $l$ by $-r+p$ for $r, p \in \mathbb{Z}$. Next we take $y=q^{\alpha+1}, \alpha>-1$, and $a q^{m}=-c b$ to get

$$
\begin{aligned}
& \sum_{k=-\infty}^{\infty} \frac{q^{k(\alpha+1)}\left(q^{k+p+1} ; q\right)_{\infty}}{\left(-c q^{k} ; q\right)_{\infty}}{ }_{1} \varphi_{1}\left(\begin{array}{c}
-c q^{k} \\
\left.q^{k+p+1} ; q, q^{\alpha+1}\right)
\end{array}\right. \\
& \quad \times\left(q^{k+r+1} ; q\right)_{\infty 1} \varphi_{1}\left(\begin{array}{c}
d q^{k+r-\alpha} \\
q^{k+r+1}
\end{array} ; q, q^{\alpha+1}\right) \\
& = \begin{cases}0, & \text { for } p<r, \\
q^{-p(\alpha+1)} d^{p-r} \frac{\left(-c q^{\alpha-p+1} / d ; q\right)_{p-r}\left(d, q^{p-r+1}, q ; q\right)_{\infty}}{\left(-c q^{-p}\right.}, & \text { for } p \geq r)_{\infty}\end{cases}
\end{aligned}
$$

Using (2.7), we see that this reduces to the second equation of (4.3) after specializing $d=-c q^{\alpha-r}$ since $\left(q^{1+r-p} ; q\right)_{p-r}=\delta_{r, p}$ for $p \geq r$. So we have proved (4.3).

\section{Proof of the dual orthogonality relations}

In this section, we prove the dual orthogonality relations of Corollary 4.2 by using a rigorous limit transition from the big $q$-Jacobi polynomials to the $q$-Bessel functions under consideration, which we now will call big q-Bessel functions. The orthogonality relations for the big $q$-Jacobi polynomials tend to the dual orthogonality relations given in Corollary 4.2. This then gives, together with the analytic proofs of the previous section, a complete alternative analytic proof of Theorem 4.1.

For $\alpha>-1, c>0, k \in \mathbb{Z}, x \in \mathbb{R}$, put

$$
\begin{aligned}
\mathcal{J}_{\alpha, k}^{c}(x ; q) & :={ }_{1} \varphi_{1}\left(\begin{array}{c}
x^{-1} \\
q^{\alpha+1} ; q,-c^{-1} x q^{k+\alpha+2}
\end{array}\right) \\
& =\left(-c^{-1} q^{k+1} ; q\right)_{\infty} \varphi_{1}\left(\begin{array}{c}
q^{\alpha+1} x, 0 \\
q^{\alpha+1} ; q,-c^{-1} q^{k+1}
\end{array}\right) .
\end{aligned}
$$

Here we used, for the second identity, the tranformation

$$
{ }_{1} \varphi_{1}\left(\begin{array}{l}
a \\
c
\end{array} ; q, x\right)=(a x / c ; q)_{\infty} \varphi_{2}\left(\begin{array}{c}
c / a, 0 \\
c
\end{array} ; q, a x / c\right)
$$

which is a limit case of Heine's transformation formula [7, (1.4.6)]. Then formulas (4.1) and (4.2) can be rewritten as

$$
L_{n}^{(\alpha)}\left(c^{-1} q^{k+1} ; q\right)=\frac{\left(q^{\alpha+1} ; q\right)_{n}}{(q ; q)_{n}} \mathcal{J}_{\alpha, k}^{c}\left(q^{n} ; q\right), \quad n \in \mathbb{Z}_{\geq 0}
$$

and

$$
M_{p}^{\left(\alpha ; c^{-1}\right)}\left(c^{-1} q^{k+1} ; q\right)=\frac{\left(q^{\alpha+1} ; q\right)_{\infty}}{\left(q,-c^{-1} q^{\alpha+1} ; q\right)_{\infty}} \mathcal{J}_{\alpha, k}^{c}\left(-c q^{p-\alpha} ; q\right), \quad p \in \mathbb{Z},
$$

respectively. 
The (dual) orthogonality relations in Corollary 4.2 can be rewritten by substitution of (4.5), (6.3), and (6.1). We obtain for $k, l \in \mathbb{Z}$ :

$$
\begin{aligned}
\sum_{n=0}^{\infty}\left(\mathcal{J}_{\alpha, k}^{c} \mathcal{J}_{\alpha, l}^{c}\right) & \left(q^{n} ; q\right) \frac{q^{n}\left(q^{n+1} ; q\right)_{\infty}}{\left(q^{n+\alpha+1} ; q\right)_{\infty}} \\
& +\sum_{p=-\infty}^{\infty}\left(\mathcal{J}_{\alpha, k}^{c} \mathcal{J}_{\alpha, l}^{c}\right)\left(-c q^{p-\alpha-1} ; q\right) \frac{c q^{p-\alpha-1}\left(-c q^{p-\alpha} ; q\right)_{\infty}}{\left(-c q^{p} ; q\right)_{\infty}} \\
= & \delta_{k, l} q^{-k(\alpha+1)} \frac{(q ; q)_{\infty}^{2}}{\left(q^{\alpha+1} ; q\right)_{\infty}^{2}} \frac{\left(-c q^{-\alpha-1},-q^{\alpha+2} c^{-1},-q^{k+1} c^{-1} ; q\right)_{\infty}}{\left(-c,-q c^{-1} ; q\right)_{\infty}}
\end{aligned}
$$

We will show that the orthogonality relations (6.5) can be obtained rigorously as a limit case of the orthogonality relations for big $q$-Jacobi polynomials.

Let $0<a<q^{-1}, b>-q^{-1}, c>0$. The big q-Jacobi polynomials are defined by

$$
P_{k}(x ; a, b,-c ; q):={ }_{3} \varphi_{2}\left(\begin{array}{c}
q^{-k}, a b q^{k+1}, x \\
a q,-c q
\end{array} ; q, q\right), \quad x \in \mathbb{R}, k \in \mathbb{Z}_{\geq 0} .
$$

Formally, we have

$$
\lim _{r \rightarrow \infty} P_{r-k}\left(q^{\alpha+1} x ; q^{\alpha}, b,-c q^{-r-1} ; q\right)=\frac{1}{\left(-c^{-1} q^{k+1} ; q\right)_{\infty}} \mathcal{J}_{\alpha, k}^{c}(x ; q) .
$$

(Substitute (6.6) and (6.2) and take termwise limits in the ${ }_{3} \varphi_{2}$.) The orthogonality relations for big $q$-Jacobi polynomials are given by

$$
\begin{aligned}
\int_{-c q}^{a q}\left(P_{k} P_{l}\right)(x ; a, b, & -c ; q) \frac{(x / a,-x / c ; q)_{\infty}}{(x,-b x / c ; q)_{\infty}} d_{q} x \\
& =\delta_{k, l} M \frac{1-a b q}{1-a b q^{2 k+1}} \frac{(q, b q,-a b q / c ; q)_{k}}{(a b q, a q,-c q ; q)_{k}}\left(a c q^{2}\right)^{k} q^{k(k-1) / 2}
\end{aligned}
$$

where

$$
M:=\frac{(1-q) a q\left(q,-c / a,-a q / c, a b q^{2} ; q\right)_{\infty}}{(a q, b q,-c q,-a b q / c ; q)_{\infty}} .
$$

See $[7,(7.3 .12)-(7.3 .14)]$. (Note the error on the right-hand side of $[7,(7.3 .13)]$ : the factor $(-a c)^{n}$ must be replaced by $\left(-a c q^{2}\right)^{-n}$.)

Formally, the orthogonality relations (6.5) can be obtained as a limit case of the orthogonality relations (6.8) for big $q$-Jacobi polynomials. In (6.8), just replace $k$ by $r-k, l$ by $r-l, c$ by $c q^{r-1}$, and $a$ by $q^{\alpha}$, and let $r \rightarrow \infty$. Because of these limit results, we call the functions $x \mapsto \mathcal{J}_{\alpha, k}^{c}(x ; q)$ big $q$-Bessel functions.

For $b=0$, we can transform the right-hand side of (6.6) by the transformation formula [7, Exercise 1.15(i)]:

$$
\tilde{P}_{k}(x ; a, 0,-c ; q):=\left(-c^{-1} q^{-k} ; q\right){ }_{k} P_{k}(x ; a, 0,-c ; q)={ }_{2} \varphi_{1}\left(\begin{array}{c}
q^{-k}, a q / x \\
a q
\end{array} ; q,-x / c\right) .
$$

Formula (6.7) for $b=0$ also can be obtained as a formal termwise limit by substituting (6.9) and (6.1) and by taking termwise limits in the ${ }_{2} \varphi_{1}$. 
Fix $\alpha>-1, c>0$, and let $r \in \mathbb{Z}_{\geq 0}$. From (6.8), we get orthogonality relations for the functions $x \mapsto \tilde{P}_{r-k}\left(q^{\alpha+1} x ; q^{\alpha}, 0,-c q^{-r-1} ; q\right)(k=r, r-1, \ldots)$ :

$$
\begin{aligned}
& \sum_{n=0}^{\infty}\left(\tilde{P}_{r-k} \tilde{P}_{r-l}\right)\left(q^{n+\alpha+1} ; q^{\alpha}, 0,-c q^{-r-1} ; q\right) \frac{q^{n}\left(q^{n+1},-c^{-1} q^{\alpha+n+r+2} ; q\right)_{\infty}}{\left(q^{\alpha+n+1} ; q\right)_{\infty}} \\
& \quad+\sum_{p=-r}^{\infty}\left(\tilde{P}_{r-k} \tilde{P}_{r-l}\right)\left(-c q^{p} ; q^{\alpha}, 0,-c q^{-r-1} ; q\right) \frac{c q^{p-\alpha-1}\left(-c q^{p-\alpha}, q^{p+r+1} ; q\right)_{\infty}}{\left(-c q^{p} ; q\right)_{\infty}} \\
& \quad=\delta_{k, l} \frac{\left(q,-c q^{-r-\alpha-1},-q^{\alpha+r+2} / c ; q\right)_{\infty}}{\left(q^{\alpha+1},-c q^{-r} ; q\right)_{\infty}} \frac{\left(q,-c^{-1} q^{k+1} ; q\right)_{r-k}}{\left(q^{\alpha+1} ; q\right)_{r-k}} q^{(\alpha+1)(r-k)}
\end{aligned}
$$

Here $k, l=r, r-1, \ldots$. Note that the orthogonality relations (6.5) and (6.10) have the same structure. We want to show that we can take a rigorous limit for $r \rightarrow \infty$ of (6.10) which yields (6.5) preserving this structure.

Proposition 6.1. Fix $\alpha>-1, c>0, k \in \mathbb{Z}$. Then, for each $x \in \mathbb{R}$, we have the pointwise limit

$$
\lim _{r \rightarrow \infty} \tilde{P}_{r-k}\left(q^{\alpha+1} x ; q^{\alpha}, 0,-c q^{-r-1} ; q\right)=\mathcal{J}_{\alpha, k}^{c}(x ; q) .
$$

Furthermore, if $M>0$, then

$$
\begin{aligned}
\left.\begin{array}{r}
\left|\tilde{P}_{r-k}\left(q^{\alpha+1} x ; q^{\alpha}, 0,-c q^{-r-1} ; q\right)\right| \\
\left|\mathcal{J}_{\alpha, k}^{c}(x ; q)\right|
\end{array}\right\} \leq & { }_{1} \phi_{1}\left(\begin{array}{c}
-M^{-1} \\
\left.q^{\alpha+1} ; q, \frac{-q^{\alpha+k+2} M}{c}\right)
\end{array}\right. \\
& \text { for }|x| \leq M \text { and } r=k, k+1, \ldots .
\end{aligned}
$$

Proof. Write

$$
\tilde{P}_{r-k}\left(q^{\alpha+1} x ; q^{\alpha}, 0,-c q^{-r-1} ; q\right)={ }_{2} \phi_{1}\left(\begin{array}{c}
q^{-r+k}, x^{-1} \\
q^{\alpha+1}
\end{array} ; q,-\frac{q^{\alpha+r+2} x}{c}\right)=\sum_{j=0}^{\infty} t_{r}(j, x)
$$

with

$$
t_{r}(j, x):=\frac{\left(q^{-r+k}, x^{-1} ; q\right)_{j}}{\left(q^{\alpha+1}, q ; q\right)_{j}}\left(-c^{-1} q^{\alpha+r+2} x\right)^{j} \quad(\text { vanishing if } j>r-k) .
$$

Also write

$$
\mathcal{J}_{\alpha, k}^{c}(x ; q)={ }_{1} \phi_{1}\left(\begin{array}{c}
x^{-1} \\
q^{\alpha+1}
\end{array} ;,-c^{-1} x q^{k+\alpha+2}\right)=\sum_{j=0}^{\infty} t(j, x)
$$

with

Then

$$
t(j, x):=\frac{\left(x^{-1} ; q\right)_{j}}{\left(q^{\alpha+1}, q ; q\right)_{j}} q^{\frac{1}{2} j(j-1)}\left(c^{-1} x q^{k+\alpha+2}\right)^{j}
$$

$$
\lim _{r \rightarrow \infty} t_{r}(j, x)=t(j, x)
$$

and

$$
\left|t_{r}(j, x)\right| \leq T(j, M) \text { for } r \geq k \text { and }|x| \leq M
$$

where

$$
T(j, M):=\frac{\left(-M^{-1} ; q\right)_{j}}{\left(q^{\alpha+1}, q ; q\right)_{j}} q^{\frac{1}{2} j(j-1)}\left(c^{-1} M q^{k+\alpha+2}\right)^{j}
$$


and

$$
\sum_{j=0}^{\infty} T(j, M)={ }_{1} \phi_{1}\left(\begin{array}{c}
-M^{-1} \\
q^{\alpha+1} ; q,-c^{-1} M q^{k+\alpha+2}
\end{array}\right)<\infty .
$$

Then the limit (6.11) follows from (6.13) and (6.14) by dominated convergence.

For the proof of (6.14), we have used :

$$
\begin{aligned}
\left|\left(1-q^{-r+k+i}\right)\left(1-x^{-1} q^{i}\right) q^{r-k} x\right| & =\left|\left(q^{i}-q^{r-k}\right)\left(x-q^{i}\right)\right| \\
& \leq q^{i}\left(M+q^{i}\right)=\left(1+M^{-1} q^{i}\right) q^{i} M .
\end{aligned}
$$

for $i=0,1, \ldots, r-k-1$ and $|x| \leq M$.

For $\tilde{P}_{r-k}\left(-c q^{p} ; q^{\alpha}, 0,-c q^{-r-1} ; q\right)$ as $-r \leq p \leq-k, r \rightarrow \infty$, we need a more refined estimate in order to be able to take limits in (6.10).

Proposition 6.2. Fix $\alpha>-1, c>0, k \in \mathbb{Z} ;$ let $r \in\{k, k+1, \ldots\}$. Then, for $p \in\{-k,-k-1, \ldots\}$, we have

$$
\begin{aligned}
& \tilde{P}_{r-k}\left(-c q^{p} ; q^{\alpha}, 0,-c q^{-r-1}\right.; q)=(q ; q)_{r-k} \frac{\left(-c q^{p} ; q\right)_{-k-p}}{\left(q^{\alpha+1}, q ; q\right)_{-k-p}}\left(-c^{-1} q^{\alpha-p+1}\right)^{-k-p} \\
& \times{ }_{2} \phi_{2}\left(\begin{array}{c}
q^{-r-p},-c q^{-k} \\
q^{\alpha+1-k-p}, q^{-k-p+1} ; q,-c^{-1} q^{\alpha-k-p+r+2}
\end{array}\right)
\end{aligned}
$$

and

$$
\begin{aligned}
\mathcal{J}_{\alpha, k}^{c}\left(-c q^{p-\alpha-1} ; q\right)= & (q ; q)_{\infty} \frac{\left(-c q^{p} ; q\right)_{-p-k}}{\left(q^{\alpha+1} ; q ; q\right)_{-p-k}}\left(-c^{-1} q^{-p+\alpha+1}\right)^{-p-k} \\
& \quad{ }_{1} \phi_{2}\left(\begin{array}{c}
-c q^{-k} \\
\left.q^{-p-k+1}, q^{\alpha+1-p-k} ; q,-c^{-1} q^{-2 p-k+\alpha+2}\right)
\end{array}\right. \\
= & \lim _{r \rightarrow \infty} \tilde{P}_{r-k}\left(-c q^{p} ; q^{\alpha}, 0,-c q^{-r-1} ; q\right) .
\end{aligned}
$$

Furthermore,

$$
\begin{aligned}
& \left.\begin{array}{r}
\left|\tilde{P}_{r-k}\left(-c q^{p} ; q^{\alpha}, 0,-c q^{-r-1} ; q\right)\right| \\
\left|\mathcal{J}_{\alpha, k}^{c}\left(-c q^{p-\alpha-1} ; q\right)\right|
\end{array}\right\} \leq \frac{\left(-c q^{p} ; q\right)_{-k-p}}{\left(q^{\alpha+1}, q ; q\right)_{-k-p}}\left(c^{-1} q^{\alpha-p+1}\right)^{-k-p} \\
& \times{ }_{1} \phi_{2}\left(\begin{array}{l}
-c q^{-k} \\
q^{\alpha+1}, q
\end{array} ; q, c^{-1} q^{k+\alpha+2}\right)
\end{aligned}
$$

for $r \in\{k, k+1, \ldots\}$ and $p \in\{-k,-k-1, \ldots\}$.

Proof. By Jackson's tranformation formula [7, (1.5.4)], we have

$$
\begin{aligned}
{ }_{2} \phi_{1}\left(\begin{array}{c}
a, b \\
c
\end{array} ; q, z\right) & =\frac{(a z ; q)_{\infty}}{(z ; q)_{\infty}}{ }_{2} \phi_{2}\left(\begin{array}{l}
a, c / b \\
c, a z
\end{array} ;, b z\right) \\
& =\frac{1}{(z ; q)_{\infty}} \sum_{j=0}^{\infty} \frac{(a, c / b ; q)_{j}\left(a z q^{j} ; q\right)_{\infty}}{(c, q ; q)_{j}} q^{\frac{1}{2} j(j-1)}(-b z)^{j}
\end{aligned}
$$

Put $z:=q^{-s+1} a^{-1}$ where $s \in \mathbb{Z}_{\geq 0}$. Then $\left(a z q^{j} ; q\right)_{\infty}=\left(q^{-s+j+1} ; q\right)_{\infty}$, so in the last sum the summation will start at $j=s$. Replace the summation index $j$ by $j+s$ and write the resulting sum again as ${ }_{2} \phi_{2}$. Then we obtain for $s \in \mathbb{Z}_{\geq 0}$ :

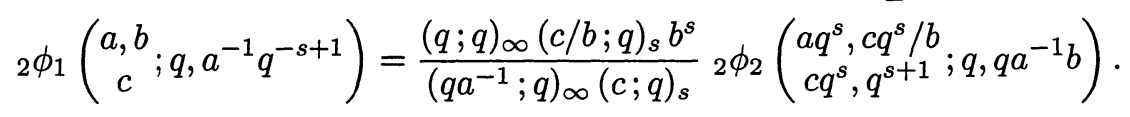


This yields (6.15) (here $s=-k-p$ ).

The first identity in (6.16) can be derived in a similar way by starting with

$$
{ }_{1} \phi_{1}\left(\begin{array}{l}
a \\
c
\end{array} ; q, z\right)=\frac{1}{(c ; q)_{\infty}} \sum_{j=0}^{\infty} \frac{(a z / c ; q)_{j}\left(q^{j} z ; q\right)_{\infty}}{(q ; q)_{j}} q^{\frac{1}{2} j(j-1)}(-c)^{j}
$$

(this is (3.5) with the right-hand side expanded) and then putting $z:=q^{-s+1}$ $\left(s \in \mathbb{Z}_{\geq 0}\right)$.

Expand the right-hand side of $(6.15)$, so

$$
\tilde{P}_{r-k}\left(-c q^{p} ; q^{\alpha}, 0,-c q^{-r-1} ; q\right)=\sum_{j=0}^{\infty} t_{r}(j ; p)
$$

with

$$
\begin{aligned}
t_{r}(j, p):= & (q ; q)_{r-k} \frac{\left(-c q^{p} ; q\right)_{-k-p}}{\left(q^{\alpha+1}, q ; q\right)_{-k-p}}\left(-c^{-1} q^{\alpha-p+1}\right)^{-k-p} \\
& \times \frac{\left(q^{-r-p},-c q^{-k} ; q\right)_{j}}{\left(q^{\alpha+1-k-p}, q^{-k-p+1}, q ; q\right)_{j}} q^{\frac{1}{2} j(j-1)}\left(c^{-1} q^{\alpha-k-p+r+2}\right)^{j}
\end{aligned}
$$

(vanishing if $j>r+p$ ). Then

$$
\left|t_{r}(j, p)\right| \leq T(j, p) \text { for } r \in\{k, k+1, \ldots\} \text { and } p \in\{-k,-k-1, \ldots\}
$$

where

$$
T(j, p):=\frac{\left(-c q^{p} ; q\right)_{-k-p}}{\left(q^{\alpha+1}, q ; q\right)_{-k-p}}\left(c^{-1} q^{\alpha-p+1}\right)^{-k-p} \frac{\left(-c q^{-k} ; q\right)_{j}}{\left(q^{\alpha+1}, q, q ; q\right)_{j}} q^{j(j-1)}\left(c^{-1} q^{k+\alpha+2}\right)^{j}
$$

and $\sum_{j=0}^{\infty} T(j, p)$ equals the right-hand side of $(6.17)$.

For the proof of (6.18), we have used

$$
\left|\left(1-q^{-r-p+i}\right) q^{r-p-k}\right|=\left|\left(q^{i}-q^{r+p}\right) q^{-2 p-k}\right| \leq q^{i} q^{k}
$$

for $i=0,1, \ldots, r+p-1$.

For fixed $p$, the limit formula in (6.16) follows from (6.11), but it also follows by taking a termwise limit for $r \rightarrow \infty$ on the right-hand side of (6.15) and by using dominated convergence in view of (6.18).

Proof of (6.5). Without loss of generality, suppose $k \geq l$. As $r \rightarrow \infty$, the right-hand side of (6.10) tends to the right-hand side of (6.5), and each term on the left-hand side of (6.10) tends to the corresponding term on the left-hand side of (6.5) (because of (6.11)). We will show that the left-hand side of (6.10) tends rigorously to the left-hand side of (6.5) by splitting up the left-hand side of (6.10) as

$$
\sum_{n=0}^{\infty}+\sum_{p=-l+1}^{\infty}+\sum_{p=-k+1}^{l}+\sum_{p=-\infty}^{-k}
$$

and by using dominated convergence for each of the three infinite sums.

As for the first sum, by $(6.12)$, the $n^{\text {th }}$ term is bounded in absolute value by $C q^{n}$ where $C>0$ and independent of $n$. Similarly, the $p^{\text {th }}$ term in the second sum is bounded in absolute value by $C q^{p}$.

By (6.17), the $p^{\text {th }}$ term in the fourth sum is bounded in absolute value by

$$
C \frac{\left(-c q^{p} ; q\right)_{-k-p}}{\left(q^{\alpha+1}, q ; q\right)_{-k-p}} \frac{\left(-c q^{p} ; q\right)_{-l-p}}{\left(q^{\alpha+1}, q ; q\right)_{-l-p}}\left(c^{-1} q^{\alpha-p+1}\right)^{-k-l-2 p} q^{p} \frac{\left(-c q^{p-\alpha} ; q\right)_{\infty}}{\left(-c q^{p} ; q\right)_{\infty}} \leq A q^{p^{2}} B^{p}
$$


where $A, B, C>0$ and independent of $p$.

Remark. As we said in the Introduction, the limit transition (6.7) from big $q$-Jacobi polynomials to big $q$-Bessel functions is inspired by what happens at a quantum group level. The regular representation of the quantum group $S U_{q}(2)$ on Podles' quantum spheres naturally decomposes into irreducible representations, generated by suitable spherical functions which turn out to depend on a single variable. Such functions thus can be identified with ordinary big $q$-Jacobi polynomials; see [22].

In the limit transition from $S U_{q}(2)$ to the Euclidean quantum group $E_{q}(2)$, quantum spheres are replaced by quantum hyperboloids, i.e., algebras in two generators $z$ and $\bar{z}$ such that $z \bar{z}=q^{2} \bar{z} z+1-q^{2}$. The corresponding regular representation decomposes into irreducible representations, each of which has an $\infty$-dimensional basis consisting of certain formal power series in $1-\bar{z} z$; see [4]. This makes it possible to identify such power series with ordinary functions, and more precisely with $q$-Bessel functions of the form $\mathcal{J}_{\alpha, k}^{c}(x ; q)$.

More precisely, the paper [4] gives in (4.9) the basis elements of the irreducible representation space as explicit formal power series $J_{r}^{(q)}$. After the substitution $\bar{z}^{j} z^{j}=$ $\left(1-\bar{z} z ; q^{-2}\right)_{j}$ (see $\left[4, \operatorname{Remark}(4.10)\right.$ (ii)]), one can identify the series $J_{r}^{(q)}$ for $\mathcal{E}=-q^{2 k}$ with $\mathcal{J}_{r, k}^{q^{2} /\left(q^{2}-1\right)^{2}}\left(1-\bar{z} z ; q^{2}\right)$ as defined by (6.1).

Acknowledgments. The first author was partly supported by C.N.R. - G.N.S.A.G.A. Work of the second author was done while at the Universiteit van Amsterdam and supported by the Netherlands Organization for Scientific Research (NWO) under project number 610.06.100.

\section{References}

1. N.I. Akhiezer, The Classical Moment Problem and Some Related Questions in Analysis, Hafner, 1965 .

2. J.M. Berezanskiŭ, Expansions in Eigenfunctions of Selfadjoint Operators, Transl. Math. Monographs 17, Amer. Math. Soc., 1968.

3. C. Berg, On some indeterminate moment problems for measures on a geometric progression, J. Comp. Appl. Math., to appear.

4. F. Bonechi, N. Ciccoli, R. Giachetti, E. Sorace, and M. Tarlini, Free q-Schrödinger equation from quantum homogeneous spaces of the 2-dim Euclidean quantum group, Comm. Math. Phys. 175 (1996), 161-176.

5. Y. Cheng, M.E.H. Ismail, and K.A. Muttalib, Asymptotics of basic Bessel functions and $q-$ Laguerre polynomials, J. Comp. Appl. Math. 54 (1994), 263-272.

6. N. Dunford and J.T. Schwartz, Linear Operators II: Spectral Theory, Interscience, 1963.

7. G. Gasper and M. Rahman, Basic Hypergeometric Series, Cambridge Univ. Press, 1990.

8. M.E.H. Ismail, The zeros of basic Bessel functions, the functions $J_{\nu+a x}(x)$, and associated orthogonal polynomials, J. Math. Anal. Appl. 86 (1982), 1-19.

9. M.E.H. Ismail, D.R. Masson, and S.K. Suslov The $q$-Bessel function on a q-quadratic grid, preprint, 1996.

10. M.E.H. Ismail and M. Rahman, The q-Laguerre polynomials and related moment problems, J. Math. Anal. Appl. 218 (1998), 155-174.

11. T. Kakehi, Eigenfunction expansion associated with the Casimir operator on the quantum group $S U_{q}(1,1)$, Duke Math. J. 80 (1995), 535-573.

12. T. Kakehi, T. Masuda, and K. Ueno, Spectral analysis of a q-difference operator which arises from the quantum $S U(1,1)$ group, J. Operator Theory 33 (1995), 159-196.

13. H.T. Koelink, $A$ basic analogue of Graf's addition formula and related formulas, Integral Transforms and Special Functions 1 (1993), 165-182. 
14. The quantum group of plane motions and the Hahn-Exton q-Bessel function, Duke Math. J. 76 (1994), 483-508.

15. - The quantum group of plane motions and basic Bessel functions, Indag. Mathem. N.S. 6 (1995), 197-211.

16. H.T. Koelink and J. Verding, Spectral analysis and the Haar functional on the quantum SU(2) group, Comm. Math. Phys. 177 (1996), 399-415.

17. T.H. Koornwinder, Jacobi functions and analysis on noncompact semisimple Lie groups. In: Special Functions: Group Theoretical Aspects and Applications, (R.A. Askey, T.H. Koornwinder, and W. Schempp, eds.), Reidel, 1984, pp.1-85.

18. T.H. Koornwinder and R.F. Swarttouw, On q-analogues of the Fourier and Hankel transforms, Trans. Amer. Math. Soc. 333 (1992), 445-461.

19. D.R. Masson and J. Repka, Spectral theory of Jacobi matrices in $\ell^{2}(\mathbb{Z})$ and the $S U(1,1)$ Lie algebra, SIAM J. Math. Anal. 22 (1991), 1131-1146.

20. T. Masuda, K. Mimachi, Y. Nakagami, M. Noumi, Y. Saburi, and K. Ueno, Unitary representations of the quantum group $S U_{q}(1,1)$ : II-Matrix elements of unitary representations and the basic hypergeometric functions, Lett. Math. Phys. 19 (1990), 195-204.

21. D.S. Moak, The q-analogue of the Laguerre polynomials, J. Math. Anal. Appl. 81 (1981), 20-47.

22. M. Noumi and K. Mimachi, Quantum 2-spheres and big q-Jacobi polynomials, Comm. Math. Phys. 128 (1990), 521-531.

23. L.L. Vaksman and L.I. Korogodskiu, An algebra of bounded functions on the quantum group of the motions of the plane and q-analogues of Bessel functions, Soviet Math. Dokl. 9 (1989), 173-177.

Dipartimento di Matematica, Università di Perugia, Via Vanvitelli 1, 06123 Perugia, Italy

E-mail: ciccoli@dipmat.unipg.it

Werkeenheid Algemene Wiskunde, Faculteit Informatietechnologie en Systemen, Postbus 5031, 2600 GA Delft, The Netherlands

E-mail: koelink@twi.tudelft.nl

Korteweg-de VRies InstituUt voor Wiskunde, Universiteit van Amsterdam, Plantage Muidergracht 24, 1018 TV AMSTERDAM, THE NetherlandS

E-mail: thk@wins.uva.nl 